\title{
Some Global Solutions of the Yang-Mills Equations in Minkowski Space
}

\author{
Robert T. Glassey ${ }^{1}$ and Walter A. Strauss ${ }^{2}$ \\ 1 Department of Mathematics, Indiana University, Bloomington, IN 47405, USA \\ 2 Department of Mathematics, Brown University, Providence, RI 02912, USA
}

\begin{abstract}
A class of global solutions of the Yang-Mills equations whose Cauchy data depend on a pair of arbitrary functions is constructed. The asymptotic propagation of the energy in space-time is studied. The same results are valid if the Yang-Mills field is coupled to a scalar field.
\end{abstract}

\section{Introduction}

This paper is concerned with solutions of the classical Yang-Mills equations in the whole of Minkowski space-time. Although one would like to exhibit such global solutions by explicit formulas, the best representation one could envisage for a general solution would be its exhibition as an infinite series or as the result of a limiting procedure. Since the equations essentially comprise a hyperbolic system of partial differential equations, it is natural to consider the Cauchy problem: do arbitrary data at a finite time (say $t=0$ ) determine a unique solution at all later and earlier times? In this paper we restrict our considerations to data which have finite energy and whose potentials vanish as $|x| \rightarrow \infty$.

It should be noted that the Yang-Mills equations are entirely different in Euclidean space. They form an elliptic, rather than a hyperbolic, system. In particular, the condition that a solution be square-integrable in space and imaginary time is a severe one. It makes the instantons form a class depending only on a few parameters.

In Sect. 2 we use a limiting procedure to construct a family of global solutions to the Yang-Mills equations (YM) if the gauge group is $S U(2)$. The solutions depend on two arbitrary functions of one variable. A substitution of the Polyakov-t'Hooft type specifies the direction of the gauge potentials $A^{\mu}$ and expresses their amplitudes in terms of a single scalar function $\alpha(|x|, t)$. The equations are thereby reduced to a single scalar nonlinear wave equation with singular coefficients. Except for the condition of finite energy, the Cauchy data for this

\footnotetext{
$\dagger$ Research supported in part by NSF Grants MCS 77-01340 and MCS 79-01965
} 
scalar equation are arbitrary. The solution is uniquely determined by such data. A critical role is played by an estimate from [3]. At the end of Sect.2 we extend this construction to the Yang-Mills equations coupled to a scalar field (YMS) with a positive self-interaction.

In Sect. 3 we consider the space-time structure of the global solutions of YM and YMS. This structure is a further consequence of the conformal invariance discussed in [2] and [3]. We begin this section with the fact that solutions propagate no faster than the speed of light. Then in Theorem 5 we state the main result on energy propagation. The energy "splits" into three components : one component $e_{\text {for }}$ carries all the energy forward to the distant future and another component $e_{\text {back }}$ carries it back to the distant past. We prove it for any nice global solution of YM or YMS and also specifically for the solutions constructed in Sect. 2. This result can also be interpreted as stating that, asymptotically as $t \rightarrow \pm \infty$, the energy of any solution propagates along light rays. For further discussion, see [4] and [1]. In particular, there can be no stationary solutions nor any solitary waves. We are continuing to assume that the potentials vanish as $|x| \rightarrow \infty$. If they do not, our asymptotic result is false: there exist stationary solutions of YMS of finite energy whose potentials approach non-zero constants as $|x| \rightarrow \infty$ (see $[7,14])$.

\section{Existence of Some Global Solutions}

We use the following notation. The physical variables are $t, x_{1}, x_{2}, x_{3}$. The derivatives are

$$
\frac{\partial}{\partial t}=\partial^{0}=\partial_{t}, \quad \frac{\partial}{\partial x_{k}}=\partial^{k} .
$$

Also $x=\left(x_{1}, x_{2}, x_{3}\right), r=|x|, \frac{\partial}{\partial r}=\partial_{r}=\sum_{k=1}^{3} \frac{x_{k}}{r} \partial^{k}$.

The potentials are $A^{\mu}(x, t)$ for $\mu=0,1,2,3$. The field strengths are

$$
\begin{aligned}
& E^{k}=\partial^{k} A^{0}+\partial^{0} A^{k}+g A^{k} \times A^{0} \quad(k=1,2,3) \\
& H^{1}=\partial^{3} A^{2}-\partial^{2} A^{3}+g A^{3} \times A^{2}
\end{aligned}
$$

with $H^{2}$ and $H^{3}$ defined by cyclic permutation of the indices. The coupling constant $g$ is real. The covariant derivatives are

$$
\begin{aligned}
& D^{k}=\partial^{k}+g A^{k} \times \quad(k=1,2,3) \\
& D^{0}=\partial^{0}-g A^{0} \times
\end{aligned}
$$

The Yang-Mills equations (YM) can then be written as

$$
\begin{aligned}
& D^{0} H^{1}=D^{3} E^{2}-D^{2} E^{3} \\
& D^{0} H^{2}=D^{1} E^{3}-D^{3} E^{1} \\
& D^{0} H^{3}=D^{2} E^{1}-D^{1} E^{2}
\end{aligned}
$$




$$
\begin{aligned}
& D^{0} E^{1}=D^{2} H^{3}-D^{3} H^{2} \\
& D^{0} E^{2}=D^{3} H^{1}-D^{1} H^{3} \\
& D^{0} E^{3}=D^{1} H^{2}-D^{2} H^{1} \\
& \sum_{k=1}^{3} D^{k} H^{k}=0 \\
& \sum_{k=1}^{3} D^{k} E^{k}=0
\end{aligned}
$$

The energy is

$$
\mathscr{E}=\frac{1}{2} \int\left(|E|^{2}+|H|^{2}\right) d x
$$

In this section we shall take the gauge group $G$ to be $S U(2)$. Its Lie algebra can be identified with Euclidean 3 -space with $\times$ being the ordinary vector product. We define

$$
\omega=x / r \quad\left(r=|x|, x \in \mathbb{R}^{3}\right),
$$

$e^{1}=(1,0,0), e^{2}=(0,1,0), e^{3}=(0,0,1)$ the standard basis and

$$
v^{k}=e^{k} \times \omega \quad(k=1,2,3)
$$

A function defined on $\mathbb{R}^{3}$ is called radial if it depends only on $r=|x|$. We define $\tilde{H}_{r}^{1}$ to be the completion of the $C^{\infty}$ functions of compact support which are radial, under the Dirichlet norm

$$
\|\nabla \phi\|_{2}=\left(\int_{\mathbb{R}^{3}}|\nabla \phi|^{2} d x\right)^{1 / 2}
$$

We define $L_{r}^{2}$ as the space of square-integrable radial functions. By $C(\mathbb{R}, X)$ we denote the space of (strongly) continuous functions on $\mathbb{R}$ with values in the space $X$.

Theorem 1. Let $\alpha_{0} \in \tilde{H}_{r}^{1}$ and $\alpha_{1} \in L_{r}^{2}$. Let $g \in \mathbb{R}$. Then there exists a unique solution of $\mathrm{YM}$ in all space and time with the following properties:

$$
\begin{aligned}
& A^{0}(x, t)=0 \\
& A^{k}(x, 0)=\alpha_{0}(r) v^{k} \quad\left(k=1,2,3 ; x \in \mathbb{R}^{3}\right) \\
& \partial_{t} A^{k}(x, 0)=\alpha_{1}(r) v^{k} \\
& A^{k} \in C\left(\mathbb{R} ; \tilde{H}_{r}^{1}\right) ; \\
& \partial_{t} A^{k} \in C\left(\mathbb{R} ; L_{r}^{2}\right) ;
\end{aligned}
$$

The energy $\mathscr{E}$ is a constant,

$$
\begin{aligned}
& A^{k}(x, t)=\alpha(r, t) v^{k} \quad(k=1,2,3), \\
& \alpha(r, t) \text { is a }(\text { real }) \text { scalar function, and } \\
& \frac{\alpha^{2}}{r}\left(g \alpha-\frac{2}{r}\right)^{2} \text { is integrable over all space-time. }
\end{aligned}
$$

In what follows we will take $g=1$ for simplicity, without loss of generality. 
Lemma 1. The inequalities

$$
\int \phi^{6} d x \leqq c\left(\int|\nabla \phi|^{2} d x\right)^{3}
$$

where $c$ is a numerical constant, and

$$
\int \phi^{2} r^{-2} d x \leqq 4 \int|\nabla \phi|^{2} d x
$$

are valid for every function $\phi \in C_{c}^{\infty}\left(\mathbb{R}^{3}\right)$ and hence for all $\phi \in \tilde{H}_{r}^{1}$. Furthermore, every $\phi \in \widetilde{H}_{r}^{1}$ is almost everywhere equal to a function which is continuous for $x \neq 0$,

$$
4 \pi r \phi^{2}(r) \leqq \int|\nabla \phi|^{2} d x
$$

and

$$
r \phi^{2}(r) \rightarrow 0 \text { as } r \rightarrow 0 \text { and } r \rightarrow \infty
$$

Proof. It suffices to prove the inequalities for $\phi \in C_{c}^{\infty}$. Equation (9) is the standard Sobolev inequality. Hardy's inequality (10) is proved as follows.

$$
\begin{aligned}
\nabla \cdot\left(x \phi^{2} / r^{2}\right) & =x \cdot 2 \phi \nabla \phi / r^{2}+\phi^{2} \nabla \cdot\left(x / r^{2}\right) \\
& =2 \phi \phi_{r} / r+\phi^{2} / r^{2}
\end{aligned}
$$

Integrating over all space, we get

$$
\begin{aligned}
\int\left(\phi^{2} / r^{2}\right) d x & =-2 \int(\phi / r) \phi_{r} d x \\
& \leqq 2\left(\int \phi^{2} / r^{2} d x\right)^{1 / 2}\left(\int \phi_{r}^{2} d x\right)^{1 / 2}
\end{aligned}
$$

from which (10) follows. Assume now that $\phi$ is radial. Integrating over the ball $\{|x|<R\}$ we have

$$
4 \pi R \phi^{2}(R) \leqq \int_{|x|<R}\left(\phi_{r}+\phi / r\right)^{2} d x
$$

Integrating instead over the exterior of a ball $\{|x|>R\}$ we have

$$
\begin{aligned}
4 \pi R \phi^{2}(R) & =\int_{|x|>R}\left[\phi_{r}^{2}-\left(\phi_{r}+\frac{1}{r} \phi\right)^{2}\right] d x \\
& \leqq \int_{|x|>R} \phi_{r}^{2} d x
\end{aligned}
$$

The continuity and (11) and (12) follow by passage to the limit.

Lemma 2. The formulas (7) and (8. $\mu$ ) reduce YM to the scalar equation

$$
\partial_{t}^{2} \alpha-\Delta \alpha+F(r, \alpha)=0
$$

where

$$
F(r, \alpha)=2 r^{-2} \alpha-3 r^{-1} \alpha^{2}+\alpha^{3}
$$


Proof. The following properties of the vectors $v^{k}$ are easy to verify:

$$
\begin{aligned}
& \sum_{k} \partial^{k} v^{k}=\sum_{k} \omega_{k} v^{k}=0 \quad\left(\omega_{k}=x_{k} / r\right) \\
& \sum_{k}\left|v^{k}\right|^{2}=2=\sum_{j, k}\left|v^{j} \times v^{k}\right|^{2} \\
& v^{k} \cdot v^{l}=\delta_{k l}-\omega_{k} \omega_{l} ; \\
& \sum_{j, k}\left|\partial^{j} v^{k}\right|^{2}=r^{-2} \\
& \sum_{j, k} \partial^{j} v^{k} \cdot v^{j} \times v^{k}=-2 r^{-1} .
\end{aligned}
$$

Since $A^{0}=0$,

$$
\begin{aligned}
E^{k} & =\partial^{0} A^{k}=\left(\partial^{0} \alpha\right) v^{k}, \text { hence } \\
\sum_{k} D^{k} E^{k} & =\sum_{k}\left[\left(\partial_{r} \partial^{0} \alpha\right) \omega_{k} v^{k}+\left(\partial^{0} \alpha\right) \partial^{k} v^{k}+\left(\partial^{0} \alpha\right) \alpha v^{k} \times v^{k}\right]=0 .
\end{aligned}
$$

This is (5.E).

We now verify (4.1). Using the definition of $H^{k}$ from (2.k) we have

$$
\begin{aligned}
& H^{2}=\frac{2 \alpha}{r} e^{2}+\left(\alpha_{r}-\frac{\alpha}{r}\right)\left(\omega_{1} v^{3}-\omega_{3} v^{1}\right)-\omega_{2} \alpha^{2} \omega, \\
& H^{3}=\frac{2 \alpha}{r} e^{3}+\left(\alpha_{r}-\frac{\alpha}{r}\right)\left(\omega_{2} v^{1}-\omega_{1} v^{2}\right)-\omega_{3} \alpha^{2} \omega .
\end{aligned}
$$

Straightforward computations then yield

$$
\begin{aligned}
& A^{2} \times H^{3}=\frac{2 \alpha^{2}}{r} v^{2} \times e^{3}+\alpha\left(\alpha_{r}-\frac{\alpha}{r}\right) \omega_{2} v^{2} \times v^{1}-\omega_{3} \alpha^{3} v^{2} \times \omega, \text { and } \\
& H^{2} \times A^{3}=\frac{-2}{r} \alpha^{2} v^{3} \times e^{2}+\alpha\left(\alpha_{r}-\frac{\alpha}{r}\right) \omega_{3} v^{3} \times v^{1}+\omega_{2} \alpha^{3} v^{3} \times \omega .
\end{aligned}
$$

When we calculate the indicated cross products, we find that

$$
A^{2} \times H^{3}+H^{2} \times A^{3}=\left(\frac{2}{r} \alpha^{2}-\alpha^{3}\right) v^{1} .
$$

These are the nonlinear terms in (4.1). The linear terms are $\partial^{2} H^{3}-\partial^{3} H^{2}$, which contribute, after a lengthy calculation, the terms

$$
\left[\alpha_{r r}+2 r^{-1} \alpha_{r}-2 r^{-2} \alpha+r^{-1} \alpha^{2}\right] v^{1} .
$$

By cyclic permutation of the indices, Eqs. (4.2) and (4.3) yield the same factors but in the directions $v^{2}$ and $v^{3}$ respectively. Thus (4.1) - (4.3) are valid if $\alpha$ satisfies the Eq. (13). The other equations in YM are automatic consequences of the definition (2.k) of $H^{k}$ (cf. [3]).

We also calculate the energy (6) in terms of $\alpha$. From the expressions for $H^{1}, H^{2}$ 
and $H^{3}$, we calculate

$$
r^{2}|H|^{2}=2 r^{2} \alpha_{r}^{2}+\left(2 \alpha-r \alpha^{2}\right)^{2}+\left(2 r \alpha^{2}\right)_{r}
$$

Therefore

$$
\mathscr{E}=\int\left[\alpha_{t}^{2}+\alpha_{r}^{2}+\frac{1}{2} \alpha^{2}\left(\frac{2}{r}-\alpha\right)^{2}\right] d x
$$

provided $r \alpha^{2}(r) \rightarrow 0$ as $r \rightarrow 0$ and $r \rightarrow \infty$.

Lemma 3. The scalar equation (13) possesses a global solution with given Cauchy data $\alpha_{0}, \alpha_{1}$.

Proof. The only difficulty in solving (13) derives from the singularity of $F(r, \alpha)$ at $r=0$. Therefore we consider the approximate equation for $\varepsilon>0$

$$
\partial_{t t} \alpha_{\varepsilon}-\Delta \alpha_{\varepsilon}+\frac{r^{2}}{r^{2}+\varepsilon} F\left(r, \alpha_{\varepsilon}\right)=0
$$

In this equation the nonlinear term is locally Lipschitz from $H^{1}$ into $L^{2}$ because of (9), (10). We choose functions $\alpha_{0 \varepsilon}(r)$ and $\alpha_{1 \varepsilon}(r)$ which belong to $C_{c}^{\infty}$ and converge to $\alpha_{0}(r)$ and $\alpha_{1}(r)$ in $\widetilde{H}_{r}^{1}$ and $L_{r}^{2}$, respectively. For fixed $\varepsilon>0$, the Eq. (13) has a unique $C^{\infty}$ solution $\alpha_{\varepsilon}(x, t)$ with compact support at fixed times with Cauchy data $\alpha_{0 \varepsilon}$ and $\alpha_{1 \varepsilon}$. This solution exists in a time interval $|t| \leqq T$. See [8] or [10].

Let $G(r, \alpha)$ be the primitive of $F(r, \alpha)$; that is, $\partial_{\alpha} G=F$ and $G(r, 0)=0$. We multiply (13) by $\partial_{t} \alpha_{\varepsilon}$ to obtain

$$
\partial_{t}\left[\frac{1}{2}\left(\partial_{t} \alpha_{\varepsilon}\right)^{2}+\frac{1}{2}\left|\nabla \alpha_{\varepsilon}\right|^{2}+\frac{r^{2}}{r^{2}+\varepsilon} G\left(r, \alpha_{\varepsilon}\right)\right]=\nabla \cdot\left[\partial_{t} \alpha_{\varepsilon} \nabla \alpha_{\varepsilon}\right] .
$$

Integrating over all space we have the energy identity

$$
\mathscr{E}_{\varepsilon}=\int\left[\frac{1}{2}\left(\partial_{t} \alpha_{\varepsilon}\right)^{2}+\frac{1}{2}\left|\nabla \alpha_{\varepsilon}\right|^{2}+\frac{r^{2}}{r^{2}+\varepsilon} G\left(r, \alpha_{\varepsilon}\right)\right] d x
$$

where $\mathscr{E}_{\varepsilon}$ is independent of time. Putting $t=0$ in this expression, we see that $\mathscr{E}_{\varepsilon}$ is determined by the initial data and so is bounded independently of $\varepsilon$. Now $G$ is a perfect square:

$$
G\left(r, \alpha_{\varepsilon}\right)=\frac{1}{4} \alpha_{\varepsilon}^{2}\left(\alpha_{\varepsilon}-\frac{2}{r}\right)^{2} \geqq 0 .
$$

Therefore the approximate solution $\alpha_{\varepsilon}$ exists for all time (see [10], [8] or [13]).

We shall also need Morawetz' radial estimate (see [6], [13] or [3]). Let $u=r \alpha_{\varepsilon}$ so that

$$
\partial_{t}^{2} u-\partial_{r}^{2} u+\left(r^{2}+\varepsilon\right)^{-1}\left(2 u-3 u^{2}+u^{3}\right)=0
$$

Multiplying this equation by $\partial_{r} u$ and integrating over $r$, we obtain the identity

$$
\frac{d}{d t} \int_{0}^{\infty} \partial_{t} u \cdot \partial_{r} u d r+\frac{1}{2}\left(\partial_{r} u(0, t)\right)^{2}+\int_{0}^{\infty} \frac{1}{2} r\left(r^{2}+\varepsilon\right)^{-2} u^{2}(u-2)^{2} d r=0
$$


since $u(0, t)=0$. Integrating over time and replacing $u$ by $r \alpha$, we obtain from the last term the bound

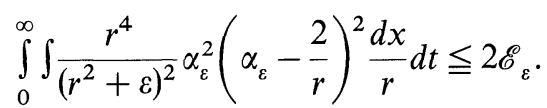

Now we can pass to the limit. There is a sequence of $\varepsilon$ 's tending to zero such that

$$
\begin{aligned}
& \alpha_{\varepsilon} \rightarrow \alpha \text { weakly* in } L^{\infty}\left(\mathbb{R}, \tilde{H}_{r}^{1}\right) \text { and } \\
& \partial_{t} \alpha_{\varepsilon} \rightarrow \partial_{t} \alpha \text { weakly* in } L^{\infty}\left(\mathbb{R}, L_{r}^{2}\right) .
\end{aligned}
$$

Therefore the derivative terms in (13) $)_{\varepsilon}$ converge in the sense of distributions. By compactness and diagonalization, the sequence may be chosen so that $\alpha_{\varepsilon} \rightarrow \alpha$ a.e. in space-time $\mathbb{R}^{4}$. So $\alpha_{\varepsilon}^{3} \rightarrow \alpha^{3}$ a.e. and $\alpha_{\varepsilon}^{3}$ is bounded in $L_{\text {loc }}^{2}\left(\mathbb{R}^{4}\right)$; hence $r^{2}(r+\varepsilon)^{-1} \alpha_{\varepsilon}^{3} \rightarrow \alpha^{3}$ weakly in $L_{\mathrm{loc}}^{2}\left(\mathbb{R}^{4}\right)$. Similarly $r^{2}\left(r^{2}+\varepsilon\right)^{-1} \alpha_{\varepsilon}^{2} \rightarrow \alpha^{2}$ weakly in $L_{\text {loc }}^{3}(\mathbb{R})$, while $r^{-1} \in L_{\text {loc }}^{3 / 2}$. Finally $r^{2}\left(r^{2}+\varepsilon\right)^{-1} \alpha_{\varepsilon} \rightarrow \alpha$ weakly in $L_{\text {loc }}^{6}\left(\mathbb{R}^{4}\right)$ while $r^{-2} \in L_{\text {loc }}^{6 / 5}$. Therefore every term in (13) $)_{\varepsilon}$ converges to the proper limit in the sense of distributions in $\mathbb{R}^{4}$. The differential equation (13) is therefore valid for $\alpha$ in all of space-time. It follows also that $\alpha$ and $\alpha_{t}$ are continuous functions of time with values in $\mathscr{D}^{\prime}\left(\mathbb{R}^{3}\right)$ and that $\alpha(x, 0)=\alpha_{0}(r)$ and $\alpha_{t}(x, 0)=\alpha_{1}(r)$.

Lemma 4. Let $f \in L_{\text {loc }}^{1}\left(\mathbb{R}, L_{r}^{2}\right), \beta_{0} \in \tilde{H}_{r}^{1}$ and $\beta_{1} \in L_{r}^{2}$. Then there is a unique solution of the linear problem

$$
\begin{gathered}
\partial_{t}^{2} \beta-\Delta \beta+2 r^{-2} \beta=f \\
\beta=\beta_{0} \text { and } \partial_{t} \beta=\beta_{1} \text { when } t=0,
\end{gathered}
$$

such that $\beta \in C\left(\mathbb{R}, \tilde{H}_{r}^{1}\right)$ and $\partial_{t} \beta \in C\left(\mathbb{R}, L_{r}^{2}\right)$ and

$$
\left.\int\left(\frac{1}{2}\left(\partial_{t} \beta\right)^{2}+\frac{1}{2}|\nabla \beta|^{2}+r^{-2} \beta^{2}\right) d x\right|_{0} ^{T}=\int_{0}^{T} \int f \cdot \partial_{t} \beta d x d t
$$

Proof. This lemma follows from the fact that the operator $-\Delta+2 r^{-2}$ restricted to $C_{c}^{\infty}\left(\mathbb{R}^{3} \backslash 0\right)$ is essentially self-adjoint $([9]$, p. 161$)$, together with the theory of linear contraction semigroups. Another method is to change variables to $\gamma=\beta / r$. Then $\gamma$ satisfies the equation

$$
\partial_{t}^{2} \gamma-\Delta_{5} \gamma=r^{-1} f
$$

where $\Delta_{5}$ is the Laplacian in 5 space dimensions. Standard facts about the inhomogenous wave equation satisfied by $\gamma$ are converted into the conclusion of Lemma 4 about $\beta$.

We now show that the solution found in Lemma 3 satisfies the properties required by Theorem 1. From (17) and Fatou's Lemma, we have

$$
\int_{0}^{\infty} \int \alpha^{2}\left(\alpha-\frac{2}{r}\right)^{2} \frac{d x}{r} d t \leqq 2 \mathscr{E}(0) .
$$

We shall apply Lemma 4 with $\beta=\alpha$ and

$$
f=\frac{3}{r} \alpha^{2}-\alpha^{3}=\frac{3}{2} \alpha\left(\frac{2}{r} \alpha-\alpha^{2}\right)+\frac{1}{2} \alpha^{3}
$$


Therefore

$$
\int f^{2} d x \leqq 3 \sup _{r}\left[r \alpha^{2}\right] \cdot \int \alpha^{2}\left(\alpha-\frac{2}{r}\right)^{2} \frac{d x}{r}+\int \alpha^{6} d x
$$

By (11) and (20) the first term on the right is integrable over time. By (9) and (6) the last term is bounded over time. Therefore $f \in L_{\text {loc }}^{1}\left(\mathbb{R}, L_{r}^{2}\right)$ and Lemma 4 is applicable.

Finally we prove uniqueness. Let $\alpha$ and $\alpha_{*}$ be two solutions with the properties listed in Theorem 1. Let $\beta=\alpha-\alpha_{*}$. Then $\beta$ statisfies Eq. (18) with

$$
f=\frac{3}{r}\left(\alpha^{2}-\alpha_{*}^{2}\right)-\left(\alpha^{3}-\alpha_{*}^{3}\right)
$$

and vanishing Cauchy data. Now

$$
f=\frac{3}{2} \beta\left(\frac{2}{r} \alpha-\alpha^{2}\right)+\frac{3}{2} \beta\left(\frac{2}{r} \alpha_{*}-\alpha_{*}^{2}\right)+\frac{1}{2} \beta^{3},
$$

so that

$$
\begin{aligned}
\int f^{2} d x & \leqq 3 \sup _{r}\left[r \beta^{2}\right] \int\left[\left(\alpha^{2}-\frac{2}{r} \alpha\right)^{2}+\left(\alpha_{*}^{2}-\frac{2}{r} \alpha_{*}\right)^{2}\right] \frac{d x}{r}+\int \beta^{6} d x \\
& \leqq(l(t)+c) \int|\nabla \beta|^{2} d x
\end{aligned}
$$

by (11), (20) and (9) where $l(t)$ is integrable and $c$ is a constant. Denoting the left side of (19) by $E(T)$ we therefore have

$$
E(T) \leqq \int_{0}^{T}(l(t)+c)^{1 / 2} E(t) d t
$$

This implies $E(T)=0$, hence $\beta=0$ and $\alpha=\alpha_{*}$.

Now (13) is invariant under time reversal. Since the solution $\alpha$ of (13) is unique, we conclude that the energy $\mathscr{E}$ in (6) $)_{\alpha}$ is in fact conserved.

We now discuss a Yang-Mills field coupled to a scalar field (cf. [3]). One introduces an additional unknown $\phi(x, t)$, a real 3-vector. When $\phi \equiv 0$ we retrieve a pure Yang-Mills field. With

the equations of motion become

$$
\psi^{\mu}=D^{\mu} \phi \quad(\mu=0,1,2,3)
$$

$$
\begin{gathered}
D^{0} E^{1}=D^{2} H^{3}-D^{3} H^{2}+g \psi^{1} \times \phi, \text { etc. } \\
\sum_{1}^{3} D^{k} E^{k}=g \psi^{0} \times \phi \\
D^{0} \psi^{0}-\sum_{1}^{3} D^{k} \psi^{k}=-V^{\prime}(\phi)
\end{gathered}
$$

Eqs. (3.k) and (5.H) remain unchanged. The equations are collectively denoted YMS. Here $V(\phi)=V_{0}\left(|\phi|^{2}\right)$ and $V^{\prime}(\phi)=2 \phi V_{0}^{\prime}\left(|\phi|^{2}\right)$ where $V_{0}^{\prime}$ is the derivative 
of the real valued function $V_{0}$. The energy is

$$
\mathscr{E}=\frac{1}{2} \int\left[|E|^{2}+|H|^{2}+\left|\psi^{0}\right|^{2}+\sum_{k=1}^{3}\left|\psi^{k}\right|^{2}+2 V(\phi)\right] d x .
$$

Theorem 2. Let $\alpha_{0}, \beta_{0} \in \tilde{H}_{r}^{1}$ and $\alpha_{1}, \beta_{1} \in L_{r}^{2}$. Let $g \in \mathbb{R}$ and $V(\phi)=c_{2}|\phi|^{2}+c_{4}|\phi|^{4}$ where $c_{2} \geqq 0, c_{4} \geqq 0(V(\phi) \equiv 0$ is allowed). Then there exists a unique solution of YMS such that

$$
A^{0}(x, t)=0, A^{k}(x, t)=\alpha(r, t) v^{k}(k=1,2,3)
$$

$$
\phi(x, t)=\beta(r, t) \omega \quad\left(\omega_{k}=x_{k} / r\right)
$$

$\alpha(x, 0)=\alpha_{0}(r), \partial_{t} \alpha(x, 0)=\alpha_{1}(r), \beta(x, 0)=\beta_{0}(r), \partial_{t} \beta(x, 0)=\beta_{1}(r)$

$\phi, A^{k} \in C\left(\mathbb{R}, \tilde{H}_{r}^{1}\right) ; \partial_{t} \phi, \partial_{t} A^{k} \in C\left(\mathbb{R}, L_{r}^{2}\right)$; the energy $\mathscr{E}$ is a constant, and

$\left\{\frac{\alpha^{2}}{r}\left(g \alpha-\frac{2}{r}\right)^{2}\right.$ and $\frac{\beta^{2}}{r}\left(g \alpha-\frac{1}{r}\right)^{2}$ and $C_{4} \beta^{4 / r}$ are integrable over all space-time.

We only give a sketch of the proof, which is similar to that of Theorem 1. The substitution $(24 . \mu),(25)$ now results in the pair of scalar equations (taking $g=1$ )

$$
\begin{aligned}
\alpha_{t t}-\Delta \alpha+\frac{2}{r^{2}} \alpha-\frac{3}{r} \alpha^{2}+\alpha^{3}+\beta^{2}\left(\alpha-\frac{1}{r}\right) & =0 \\
\beta_{t t}-\Delta \beta+\frac{2}{r^{2}} \beta+2 \alpha \beta\left(\alpha-\frac{2}{r}\right)+2 \beta V_{0}^{\prime}\left(\beta^{2}\right) & =0
\end{aligned}
$$

The energy is formally

$$
\mathscr{E}=\int\left[\alpha_{t}^{2}+\alpha_{r}^{2}+\frac{1}{2} \alpha^{2}\left(\alpha-\frac{2}{r}\right)^{2}+\frac{1}{2} \beta_{t}^{2}+\frac{1}{2} \beta_{r}^{2}+\beta^{2}\left(\alpha-\frac{1}{r}\right)^{2}+V_{0}\left(\beta^{2}\right)\right] d x .
$$

Since $V_{0} \geqq 0$ by assumption, each term in $\mathscr{E}$ is formally bounded. As in Lemma 3 we introduce the factor $r^{2}\left(r^{2}+\varepsilon\right)^{-1}$. The limiting argument works because $V_{0}$ has degree 4 . The estimates (26) come formally from Theorem 4 of [3], namely

$$
\iint\left[|H \omega|^{2}+|\Psi|^{2}-|\Psi \omega|^{2}+c_{4}|\phi|^{4}\right] \frac{1}{r} d x d t<\infty .
$$

In this case $\Psi \omega=\beta_{r} \omega$ and $|\Psi|^{2}=\beta_{r}^{2}+2 \beta^{2}\left(\alpha-r^{-1}\right)^{2}$ and $|H \omega|^{2}=\alpha^{2}\left(\alpha-\frac{2}{r}\right)^{2}$. The estimates and the uniqueness proof follow from the same techniques as before.

Remark. Theorem 2 is valid verbatim if $c_{2}<0$, except that (26) is valid only over finite time intervals, and we assume $\alpha_{0}, \beta_{0} \in L^{2}$. Indeed, in this case the energy $\mathscr{E}$ above has a negative term $-\int \phi^{2} d x$. It is estimated by

$$
\int|\phi(x, T)|^{2} d x \leqq 2 \int|\phi(x, 0)|^{2} d x+4 T \int_{0}^{T} \int\left|\partial_{t} \phi(x, t)\right|^{2} d x d t
$$

and so each term in $\mathscr{E}$ is bounded for bounded time. Minor changes are made in the rest of the proof. More generally we can state the following existence (but not uniqueness) theorem. 
Theorem 3. Assume only that $V_{0}$ is a $C^{1}$ function such that

$$
V_{0}(s) \geqq 0 \text { and } V_{0}^{\prime}(s) \geqq 0 \text { for } s \geqq s_{0} .
$$

Let $\alpha_{0}, \beta_{0}, \alpha_{1}, \beta_{1}$ be as in Theorem 2 . Then there exists a solution of YMS satisfying the conclusions of Theorem 2 except that: $\phi, A^{k}$ are weakly continuous with values in $\tilde{H}_{r}^{1} ; \partial_{t} \phi, \partial_{t} A^{k}$ are weakly continuous with values in $L_{r}^{2} ; \mathscr{E}$ satisfies the inequality $\mathscr{E}(t) \leqq \mathscr{E}(0)$; and the expressions in (26) are integrable over all space but only over finite time-intervals.

Proof. By assumption $V(\phi)$ and $\phi \cdot V^{\prime}(\phi)$ are bounded below by $O\left(|\phi|^{2}\right)$. Therefore we obtain energy bounds as in the preceding remark. We introduce the factor $r^{2} /\left(r^{2}+\varepsilon\right)$ as in Theorem 2 . We approximate $V_{0}$ by $V_{\varepsilon}$, where $V_{\varepsilon}(s)$ grows slowly for large $s$ (for each $\varepsilon$ ). The passage to the limit is effected by the method of Theorem 1 with the omission of Lemma 4 and its consequences. See [12] for the very similar case of a scalar field.

\section{Propagation of the Energy}

Theorem 4. YM is causal. YMS is causal if $V \geqq 0$.

Suppose we begin with any smooth solution of YMS. We have the law of conservation of energy

$$
\partial^{0} e=\sum_{k} \partial^{k} p^{k}
$$

with the energy density $e$ and momentum densities $p^{k}$ (see [3]). We integrate this identity over a piece of the solid light cone (see sketch) with base $B$, top $T$ and side $K$. By the divergence theorem,

$$
\int_{T} e-\int_{B} e+\frac{1}{\sqrt{2}} \int_{K}\left(\sum_{k} \omega_{k} p^{k}+e\right)=0 .
$$

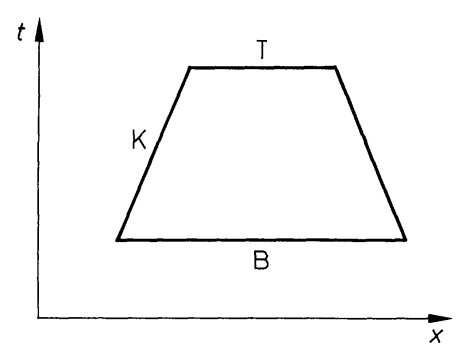

But $\left|\sum_{k} \omega_{k} p^{k}\right| \leqq e(\mathrm{cf} .[2],[3])$ so that the integral over $K$ is non-negative. Therefore

$$
\int_{T} e d x \leqq \int_{B} e d x
$$

In particular if $e=0$ on $B$, then the Cauchy data vanish in the solid cone depending on $B$. This is a precise expression of the causality.

In particular, the global solutions constructed in Sect. 2 satisfy (29). Indeed, they are defined in terms of scalar functions $\alpha$ and $\beta$, which are approximated by 
$\alpha_{\varepsilon}$ and $\beta_{\varepsilon}$. The inequality (29) is valid for the approximate solution for each $\varepsilon$, which is smooth [10]. The passage to the weak limit described in Sect. 2 implies that (29) is valid for the exact solution, provided that $B \subset\{t=0\}$, the time when the Cauchy data are prescribed. Thus (29) is valid for any of the solutions in Theorems 1, 2 and 3.

Theorem 5. (Energy splitting). Consider a solution satisfying any one of the conditions (I)-(III) below. Then there exists a decomposition of the energy density e into non-negative parts

$$
e=e_{\mathrm{for}}+e_{\mathrm{back}}+e_{\mathrm{ang}}
$$

such that

$$
\begin{gathered}
\int_{\mathbb{R}^{3}}\left(e_{\text {back }}+e_{\text {ang }}\right) d x \rightarrow 0 \quad \text { as } t \rightarrow+\infty \\
\int_{\mathbb{R}^{3}}\left(e_{\text {for }}+e_{\text {ang }}\right) d x \rightarrow 0 \text { as } t \rightarrow-\infty
\end{gathered}
$$

These three expressions are defined in the proof below; $e_{\text {for }}$ carries all the energy forward in time and $e_{\text {back }}$ carries all the energy backward in time, asymptotically. Assume that the initial data satisfy $\int\left(r^{2}+1\right) e d x<\infty$. Furthermore assume one of the following:

(I) The solution is a $C^{2}$ solution of YMS such that $\int\left(r^{2}+1\right) e d x<\infty$ at all times; $G$ is a compact Lie group and $0 \leqq 4 V(\phi) \leqq \phi \cdot V^{\prime}(\phi)$.

(II) It is a solution of YM given in Theorem 1.

(III) It is a solution of YMS given in Theorem 2, where $V(\phi)=C_{4}|\phi|^{4}, C_{4} \geqq 0$.

Proof. This result is a consequence of the conformal invariance of the equations, specifically the first inversional identity derived in [3]. It states that

$$
\int\left[\left(t^{2}+r^{2}\right) e+2 \operatorname{tr} \sum_{k} \omega_{k} p^{k}+2 t \phi \cdot \psi^{0}-\phi \cdot \phi\right] d x \leqq \text { const. }
$$

under assumption (I). Here

$$
\begin{aligned}
& p^{k}=p_{\mathrm{YM}}^{k}+\psi^{0} \cdot \psi^{k} \quad(k=1,2,3) \\
& p_{\mathrm{YM}}^{1}=H^{2} \cdot E^{3}-H^{3} \cdot E^{2} \text { etc }
\end{aligned}
$$

For the convenience of the reader we record the complete YMS system here. (See [3]).

$$
\begin{aligned}
& D^{0} H^{1}=D^{3} E^{2}-D^{2} E^{3} \\
& D^{0} H^{2}=D^{1} E^{3}-D^{3} E^{1} \\
& D^{0} H^{3}=D^{2} E^{1}-D^{1} E^{2} \\
& D^{0} E^{1}=D^{2} H^{3}-D^{3} H^{2}+g \psi^{1} \times \phi \\
& D^{0} E^{2}=D^{3} H^{1}-D^{1} H^{3}+g \psi^{2} \times \phi \\
& D^{0} E^{3}=D^{1} H^{2}-D^{2} H^{1}+g \psi^{3} \times \phi \\
& \sum D^{k} H^{k}=0
\end{aligned}
$$




$$
\begin{aligned}
& \sum D^{k} E^{k}=g \psi^{0} \times \phi \\
& D^{0} \psi^{0}-\sum D^{k} \psi^{k}=-V^{\prime}(\phi) \\
& \psi^{\mu}=D^{\mu} \phi(\mu=0,1,2,3) .
\end{aligned}
$$

As in [3] we split the integrand $I$ in (30) into the Yang-Mills part $I_{\mathrm{YM}}$ and the scalar part $I_{s}$. Thus $I=I_{\mathrm{YM}}+I_{s}$ where

$$
I_{\mathrm{YM}}=\frac{1}{2}\left(t^{2}+r^{2}\right)\left(|E|^{2}+|H|^{2}\right)+2 \operatorname{tr} \sum_{k} \omega_{k} p_{\mathrm{YM}}^{k}
$$

and

$$
I_{s}=\frac{t^{2}+r^{2}}{2}\left(\left|\psi^{0}\right|^{2}+\sum_{1}^{3}\left|\psi^{k}\right|^{2}+2 V(\phi)\right)+2 t \psi^{0} \cdot(\Psi x+\phi)-|\phi|^{2}
$$

where $\Psi$ is the matrix with the three columns $\psi^{1}, \psi^{2}, \psi^{3}$. Define $X^{k}=\psi^{k}+x_{k} r^{-2} \phi$ and let $\Xi$ be the matrix with columns $X^{1}, X^{2}, X^{3}$. We showed in [3] that

$$
|\Xi|^{2}=|\Psi|^{2}+r^{-2} \partial_{r}\left(r|\phi|^{2}\right)
$$

Hence

$$
I_{s}=I_{s}^{*}-\frac{1}{2 r^{2}} \partial_{r}\left\{\left(t^{2}+r^{2}\right) r|\phi|^{2}\right\}
$$

with

$$
I_{s}^{*}=\frac{t^{2}+r^{2}}{2}\left(\left|\psi^{0}\right|^{2}+|\Xi|^{2}+2 V(\phi)\right)+2 t \psi^{0} \cdot \Xi x
$$

Now consider $I_{\mathrm{YM}}$. We define $(E, H)=\operatorname{tr}\left(E^{T} H\right)$ so that $|E|^{2}=(E, E)$. Here $E^{T}$ is the transpose of $E$. We further define $\omega \times E$ as the matrix with columns $\omega_{2} E^{3}-$ $\omega_{3} E^{2}, \omega_{3} E^{1}-\omega_{1} E^{3}, \omega_{1} E^{2}-\omega_{2} E^{1}$, respectively and note the elementary identity

$$
|E|^{2}=|E \omega|^{2}+|\omega \times E|^{2} .
$$

With this notation, we can write

$$
\begin{aligned}
I_{\mathrm{YM}}= & \frac{r^{2}+t^{2}}{2}\left(|E|^{2}+|H|^{2}\right)+2 \operatorname{tr} \sum_{1}^{3} \omega_{k} p_{\mathrm{YM}}^{k} \\
= & \frac{1}{2}\left(r^{2}+t^{2}\right)\left(|E|^{2}+|H|^{2}\right)+r t(E, \omega \times H)-r t(H, \omega \times E) \\
= & \frac{1}{4}\left(r^{2}+t^{2}\right)\left(|E \omega|^{2}+|H \omega|^{2}\right)+\frac{1}{8}(r+t)^{2}\left[|E+\omega \times H|^{2}\right. \\
& \left.+|H-\omega \times E|^{2}\right]+\frac{1}{8}(r-t)^{2}\left[|E-\omega \times H|^{2}+|H+\omega \times E|^{2}\right]
\end{aligned}
$$

For the computation of $I_{s}^{*}$, we add the elementary identities

$$
\frac{1}{4}(r \pm t)^{2}\left|\psi^{0} \pm \Xi \omega\right|^{2}=\frac{1}{4}\left(r^{2} \pm 2 r t+t^{2}\right)\left[\left|\psi^{0}\right|^{2}+|\Xi \omega|^{2} \pm 2 \psi^{0} \cdot \Xi \omega\right]
$$

to get

$$
\begin{aligned}
& \frac{1}{4}(r+t)^{2}\left|\psi^{0}+\Xi \omega\right|^{2}+\frac{1}{4}(r-t)^{2}\left|\psi^{0}-\Xi \omega\right|^{2} \\
& \quad=\frac{1}{2}\left(r^{2}+t^{2}\right)\left(\left|\psi^{0}\right|^{2}+|\Xi \omega|^{2}\right)+2 r t \psi^{0} \cdot \Xi \omega \\
& \quad=I_{s}^{*}-\frac{\left(r^{2}+t^{2}\right)}{2}\left[|\Xi|^{2}-|\Xi \omega|^{2}+2 V(\phi)\right] .
\end{aligned}
$$


Using (31), (32) and the original decomposition

$$
I=I_{\mathrm{YM}}+I_{s}=I_{\mathrm{YM}}+I_{s}^{*}-\frac{1}{2 r^{2}} \partial_{r}\left\{\left(r^{2}+t^{2}\right) r|\phi|^{2}\right\}
$$

we can write

$$
\begin{aligned}
I= & \frac{r^{2}+t^{2}}{4}\left[|E \omega|^{2}+|H \omega|^{2}+2\left(|\Xi|^{2}-|\Xi \omega|^{2}+2 V(\phi)\right)\right] \\
& +\frac{(r+t)^{2}}{8}\left[|E+\omega \times H|^{2}+|H-\omega \times E|^{2}+2\left|\psi^{0}+\Xi \omega\right|^{2}\right] \\
& +\frac{(r-t)^{2}}{8}\left[|E-\omega \times H|^{2}+|H+\omega \times E|^{2}+2\left|\psi^{0}-\Xi \omega\right|^{2}\right] \\
& -\frac{1}{2 r^{2}} \partial_{r}\left\{\left(r^{2}+t^{2}\right) r|\phi|^{2}\right\} .
\end{aligned}
$$

If we define

$$
\begin{aligned}
& 4 e_{\text {ang }}=|E \omega|^{2}+|H \omega|^{2}+2\left(|\Xi|^{2}-|\Xi \omega|^{2}\right)+4 V(\phi) ; \\
& 8 e_{\text {back }}=|E+\omega \times H|^{2}+|H-\omega \times E|^{2}+2\left|\psi^{0}+\Xi \omega\right|^{2} ; \\
& 8 e_{\text {for }}=|E-\omega \times H|^{2}+|H+\omega \times E|^{2}+2\left|\psi^{0}-\Xi \omega\right|^{2},
\end{aligned}
$$

then (33) implies directly that these expressions have the properties claimed. This completes the proof of Theorem 5 under Assumption (I).

In order to prove Theorem 5 under Assumption (III), our main task is to derive the analogue of the first inversional identity (30) for the approximate equations. Then we shall pass to the limit. Note that (II) is just the special case of (III) when $\phi \equiv 0$. From Sect. 2 the equations for $\alpha, \beta$ are

$$
\begin{aligned}
& \alpha_{t t}-\Delta \alpha+F_{1}(r, \alpha, \beta)=0 \\
& \beta_{t t}-\Delta \beta+F_{2}(r, \alpha, \beta)=0
\end{aligned}
$$

where

$$
\begin{aligned}
& F_{1}(r, \alpha, \beta)=\frac{2}{r^{2}} \alpha-\frac{3}{r} \alpha^{2}+\alpha^{3}+\beta^{2}\left(\alpha-\frac{1}{r}\right) \\
& F_{2}(r, \alpha, \beta)=\frac{2}{r^{2}} \beta+2 \alpha \beta\left(\alpha-\frac{2}{r}\right)+2 \beta V_{0}^{\prime}\left(\beta^{2}\right)
\end{aligned}
$$

We consider the approximate equations

$$
\begin{aligned}
& \alpha_{t t}-\Delta \alpha+\frac{r^{2}}{r^{2}+\varepsilon} F_{1}(r, \alpha, \beta)=0 \\
& \left.\beta_{t t}-\Delta \beta+\frac{r^{2}}{r^{2}+\varepsilon} F_{2}(r, \alpha, \beta)=0\right)
\end{aligned}
$$

with solutions $\alpha=\alpha_{\varepsilon}, \beta=\beta_{\varepsilon}$. We assume that the Cauchy data of $\alpha_{\varepsilon}, \beta_{\varepsilon}$ are smooth and have compact support. From Sect. 2 we know that the energy density of the 
approximate system is given by

$$
\begin{aligned}
e_{\varepsilon}= & \frac{1}{2}\left(\alpha_{t}^{2}+\alpha_{r}^{2}\right)+\frac{1}{4}\left(\beta_{t}^{2}+\beta_{r}^{2}\right) \\
& +\frac{r^{2}}{r^{2}+\varepsilon}\left[\frac{1}{4} \alpha^{2}\left(\alpha-\frac{2}{r}\right)^{2}+\frac{1}{2} \beta^{2}\left(\alpha-\frac{1}{r}\right)^{2}+\frac{1}{2} V_{0}\left(\beta^{2}\right)\right]
\end{aligned}
$$

where $\alpha=\alpha_{\varepsilon}, \beta=\beta_{\varepsilon}$.

We now derive the inversional identity for the approximate equations. This tedious calculation will only be sketched, since it is a special case of the derivation of (30), modified by the introduction of the factor $r^{2} /\left(r^{2}+\varepsilon\right)($ see [3], [13]).

We multiply the approximate equation $(27)_{\varepsilon}$ for $\alpha=\alpha_{\varepsilon}$ by

$$
\left(r^{2}+t^{2}\right) \alpha_{t}+2 \operatorname{tr} \alpha_{r}+2 t \alpha
$$

and use the multiplier

$$
\frac{1}{2}\left(r^{2}+t^{2}\right) \beta_{t}+\operatorname{tr} \beta_{r}+t \beta
$$

on Eq. (28) . These expressions are added and the result is integrated over $\mathbb{R}^{3}$ (with usual Lebesgue measure $d x=4 \pi r^{2} d r$ ). We obtain in this manner the identity

$$
\begin{aligned}
& \frac{d}{d t} \int_{\mathbb{R}^{3}}\left[( r ^ { 2 } + t ^ { 2 } ) \left(\frac{1}{2} \alpha_{t}^{2}+\frac{1}{2} \alpha_{r}^{2}+\frac{1}{4} \beta_{t}^{2}+\frac{1}{4} \beta_{r}^{2}+2 r t \alpha_{t} \alpha_{r}+r t \beta_{t} \beta_{r}\right.\right. \\
& \left.\quad+2 t \alpha \alpha_{t}+t \beta \beta_{t}-\alpha^{2}-\frac{1}{2} \beta^{2}\right] d x \\
& \quad+\int_{\mathbb{R}^{3}} \frac{r^{2}\left(r^{2}+t^{2}\right)}{r^{2}+\varepsilon}\left[\alpha_{t} F_{1}+\frac{1}{2} \beta_{t} F_{2}\right] d x \\
& \quad+\int_{\mathbb{R}^{3}} \frac{r^{3} t}{r^{2}+\varepsilon}\left[2 \alpha_{r} F_{1}+\beta_{r} F_{2}\right] d x \\
& \quad+\int_{\mathbb{R}^{3}} \frac{r^{2} t}{r^{2}+\varepsilon}\left[2 \alpha F_{1}+\beta F_{2}\right] d x=0 .
\end{aligned}
$$

Straightforward use of (34), (35) then gives us

$$
\alpha_{t} F_{1}+\frac{1}{2} \beta_{t} F_{2}=\frac{\partial}{\partial t}\left[\frac{\alpha^{2}}{r^{2}}-\frac{\alpha^{3}}{r}+\frac{\alpha^{4}}{4}+\frac{\alpha^{2} \beta^{2}}{2}-\frac{\alpha \beta^{2}}{r}+\frac{\beta^{2}}{2 r^{2}}+\frac{1}{2} V_{0}\left(\beta^{2}\right)\right]
$$

and

$$
2 \alpha F_{1}+\beta F_{2}=\frac{4 \alpha^{2}}{r^{2}}+\frac{2 \beta^{2}}{r^{2}}-\frac{6 \alpha^{3}}{r}+2 \alpha^{4}+4 \alpha^{2} \beta^{2}-\frac{6 \alpha \beta^{2}}{r}+2 \beta^{2} V_{0}^{\prime}\left(\beta^{2}\right) .
$$

Similarly, we find

$$
\begin{aligned}
2 \alpha_{r} F_{1}+\beta_{r} F_{2}=\frac{\partial}{\partial r} & {\left[\frac{2 \alpha^{2}}{r^{2}}-\frac{2 \alpha^{3}}{r}+\frac{\alpha^{4}}{2}-\frac{2}{r} \alpha \beta^{2}+\frac{\beta^{2}}{r^{2}}+\alpha^{2} \beta^{2}+V_{0}\left(\beta^{2}\right)\right] } \\
& +\frac{4}{r^{3}} \alpha^{2}-\frac{2}{r^{2}} \alpha^{3}-\frac{2}{r^{2}} \alpha \beta^{2}+\frac{2}{r^{3}} \beta^{2} .
\end{aligned}
$$


Using this we can compute the third term in (37) by integrating by parts. The result is

$$
\begin{aligned}
& \int_{\mathbb{R}^{3}} \frac{r^{3} t}{r^{2}+\varepsilon}\left(2 \alpha_{r} F_{1}+\beta_{r} F_{2}\right) d x \\
& =\int_{\mathbb{R}^{3}} \frac{r^{2} t}{r^{2}+\varepsilon}\left[-\frac{2 \alpha^{2}}{r^{2}}+\frac{4 \alpha^{3}}{r}-\frac{\beta^{2}}{r^{2}}+\frac{4 \alpha \beta^{2}}{r}-\frac{3 \alpha^{4}}{2}-3 \alpha^{2} \beta^{2}-3 V_{0}\left(\beta^{2}\right)\right] d x \\
& \quad-2 \varepsilon \int_{\mathbb{R}^{3}} \frac{r^{2} t}{\left(r^{2}+\varepsilon\right)^{2}}\left[\frac{2 \alpha^{2}}{r^{2}}-\frac{2 \alpha^{3}}{r}+\frac{\alpha^{4}}{2}-\frac{2}{r} \alpha \beta^{2}+\frac{\beta^{2}}{r^{2}}+\alpha^{2} \beta^{2}+V_{0}\left(\beta^{2}\right)\right] d x
\end{aligned}
$$

We now combine these computations in (37) to obtain the first inversional identity:

where

$$
\frac{d}{d t} \int I d x=J_{\varepsilon} \equiv 2 \varepsilon t \int \frac{r^{2}}{\left(r^{2}+\varepsilon\right)^{2}} Q d x
$$

$$
Q=\frac{1}{2} \alpha^{2}\left(\alpha-\frac{2}{r}\right)^{2}+\beta^{2}\left(\alpha-\frac{1}{r}\right)^{2}+V_{0}\left(\beta^{2}\right)
$$

and

$$
\begin{aligned}
I= & \frac{1}{2}\left(r^{2}+t^{2}\right)\left(\alpha_{t}^{2}+\alpha_{r}^{2}+\frac{1}{2}\left(\beta_{t}^{2}+\beta_{r}^{2}\right)\right) \\
& +r t\left(2 \alpha_{t} \alpha_{r}+\beta_{t} \beta_{r}\right)+t\left(2 \alpha \alpha_{t}+\beta \beta_{t}\right)-\alpha^{2}-\frac{1}{2} \beta^{2} \\
& +\frac{r^{2}\left(r^{2}+t^{2}\right)}{2\left(r^{2}+\varepsilon\right)} Q .
\end{aligned}
$$

We keep in mind that $\alpha=\alpha_{\varepsilon}$ and $\beta=\beta_{\varepsilon}$ here. Now the analogue of estimate (17) in the case of YMS is

$$
\int_{0}^{\infty} \int \frac{r^{3}}{\left(r^{2}+\varepsilon\right)^{2}} Q\left(\alpha_{\varepsilon}, \beta_{\varepsilon}\right) d x d t \leqq \text { constant }
$$

We use this to estimate $J_{\varepsilon}$ as follows. Let $\delta=\varepsilon^{3 / 4}$. Then

$$
\begin{aligned}
A \equiv \int_{0}^{T} 2 \varepsilon t \int_{r>\delta} \frac{r^{2}}{\left(r^{2}+\varepsilon\right)^{2}} Q d x d t & \leqq 2 \varepsilon^{1 / 4} T \int_{0}^{T} \frac{r^{3}}{\left(r^{2}+\varepsilon\right)^{2}} Q d x d t \\
& \leqq 2 \varepsilon^{1 / 4} T c .
\end{aligned}
$$

On the other hand,

$$
B \equiv \int_{0}^{T} 2 \varepsilon t \int_{r>\delta} \frac{r^{2}}{\left(r^{2}+\varepsilon\right)^{2}} Q d x d t \leqq \frac{1}{\varepsilon} T^{2} \sup _{t} \int_{r<\delta} r^{2} Q d x
$$

Recalling (11) and the definition of $Q$, we have $Q \leqq c r^{-3}$ (for $r<1$ ). Hence

$$
B \leqq c \varepsilon^{-1} T^{2} \int_{0}^{\delta} r^{2} r^{-3} r^{2} d r \leqq c \varepsilon^{-1} T^{2} \delta^{2}=c T^{2} \sqrt{\varepsilon}
$$

Therefore, for any $T$,

$$
\int_{0}^{T} J_{\varepsilon}(t) d t \rightarrow 0 \quad \text { as } \quad \varepsilon \rightarrow 0
$$


It follows from (38) that

$$
\left.\lim _{\varepsilon \rightarrow 0} \int I\left(\alpha_{\varepsilon}, \beta_{\varepsilon}\right)\right|_{t=T} d x=\left.\lim _{\varepsilon \rightarrow 0} \int I\left(\alpha_{\varepsilon}, \beta_{\varepsilon}\right)\right|_{t=0} d x .
$$

As in (33), we can express I essentially as a sum of squares as follows:

$$
\begin{aligned}
I\left(\alpha_{\varepsilon}, \beta_{\varepsilon}\right)= & \frac{1}{4}(r+t)^{2}\left[\partial_{t} \alpha_{\varepsilon}+\frac{1}{r} \partial_{r}\left(r \alpha_{\varepsilon}\right)\right]^{2}+\frac{1}{4}(r-t)^{2}\left[\partial_{t} \alpha_{\varepsilon}-\frac{1}{r} \partial_{r}\left(r \alpha_{\varepsilon}\right)\right]^{2} \\
& +\frac{1}{8}(r+t)^{2}\left[\partial_{t} \beta_{\varepsilon}+\frac{1}{r} \partial_{r}\left(r \beta_{\varepsilon}\right)\right]^{2}+\frac{1}{8}(r-t)^{2}\left[\partial_{t} \beta_{\varepsilon}-\frac{1}{r} \partial_{r}\left(r \beta_{\varepsilon}\right)\right]^{2} \\
& +\frac{r^{2}\left(r^{2}+t^{2}\right)}{2\left(r^{2}+\varepsilon\right)}\left[\frac{1}{2} \alpha_{\varepsilon}^{2}\left(\alpha_{\varepsilon}-\frac{2}{r}\right)^{2}+\beta_{\varepsilon}^{2}\left(\alpha_{\varepsilon}-\frac{1}{r}\right)^{2}+V_{0}\left(\beta_{\varepsilon}^{2}\right)\right] \\
& -\frac{1}{2 r^{2}} \partial_{r}\left[\left(t^{2}+r^{2}\right)\left(r \alpha_{\varepsilon}^{2}+\frac{1}{2} r \beta_{\varepsilon}^{2}\right)\right]
\end{aligned}
$$

The last term can be dropped because it integrates to zero. The right side of (40) depends only on the initial data and can be chosen to converge to $\int I(\alpha, \beta) d x$ at $t=0$. Now fix a time $T$. Each of the expressions

and

$$
(r \pm T)\left[\partial_{t} \alpha_{\varepsilon} \pm \frac{1}{r} \partial_{r}\left(r \alpha_{\varepsilon}\right)\right]
$$

$$
(r \pm T)\left[\partial_{t} \beta_{\varepsilon} \pm \frac{1}{r} \partial_{r}\left(r \beta_{\varepsilon}\right)\right],
$$

at time $T$ converges in the sense of distributions on $\mathbb{R}^{3}$ to the same expression with $\varepsilon$ omitted, as we can see from the proofs in Sect. 2 . Therefore (40) and (41) imply that each of them converges weakly in $L^{2}\left(\mathbb{R}^{3}\right)$ to the same limit. Together with the almost everywhere convergence of the last term in (41), this implies that

$$
\left.\int I(\alpha, \beta)\right|_{t=T} d x \leqq\left.\liminf _{\varepsilon \rightarrow 0} \int I\left(\alpha_{\varepsilon}, \beta_{\varepsilon}\right)\right|_{t=T} d x=\left.\int I(\alpha, \beta)\right|_{t=0} d x
$$

This is exactly the integrated form of (33) for our special solutions. The conclusion follows immediately.

These results can be applied as in [4] to establish the nonexistence of solitary wave solutions (including those of speed one).

Corollary. Under the assumptions of Theorem 5, $\int\left[|E \omega|^{2}+|H \omega|^{2}\right] d x=0\left(t^{-2}\right)$. If $A^{0} \equiv 0$, then $\left.\int|A \omega|^{2} d x=0(\log |t|)^{2}\right)$ as $t \rightarrow \pm \infty$.

Proof. The first statement is obvious since $|E \omega|^{2}+|H \omega|^{2} \leqq 4 e_{\text {ang }}$. By a gauge transformation, the "temporal gauge condition" $A^{0} \equiv 0$ can always be assumed. Then $E^{k}=\partial^{0} A^{k}$ so that $E \omega=\partial^{0} A \omega$ and

$$
\frac{d}{d t} \int|A \omega|^{2} d x=\int A \omega \cdot E \omega d x \leqq c\left(\left(l+t^{2}\right)^{-1} \int|A \omega|^{2} d x\right)^{1 / 2}
$$

from which the Corollary follows. 


\section{References}

1. Costa, D., Strauss, W. : Energy Splitting. Q. Appl. Math. (to appear)

2. Glassey, R., Strauss, W. : Commun. Math. Phys. 65, 1-13 (1979)

3. Glassey, R., Strauss, W. : Commun. Math. Phys. 67, 51-67 (1979)

4. Glassey, R., Strauss, W. : Propagation of the energy of Yang-Mills fields. In: Bifurcation Phenomena in Mathematical Physics and Related Topics pp. 231-241. Dordrecht: Reidel Publishing Co., 1980

5. Moncrief, V. : Global existence of Maxwell-Klein-Gordon fields in $(2+1)$-dimensional spacetime. (to appear)

6. Morawetz, C. S. : Proc. R. Soc. A306, 291-296 (1968)

7. Prasad, M., Sommerfield, C. : Phys. Rev. Lett. 35, 760-762 (1975)

8. Reed, M. : Abstract nonlinear wave equations. In: Lecture Notes in Mathematics, Vol. 507. Berlin, Heidelberg, New York : Springer 1976

9. Reed, M., Simon, B. : Methods of modern mathematical physics, Vol. II. New York: Academic Press 1975

10. Segal, I. E. : Ann. Math. 78, 339-364 1963

11. Segal, I. E. : J. Funct. Anal. 33, 175-194 1979

12. Strauss, W.: Anais. Acad. Bras. Cienc. 42, 645-651 (1970)

13. Strauss, W. : Nonlinear invariant wave equations. In : Lecture Notes in Physics, Vol. 73, pp. 197-249 (Erice, 1977). Berlin, Heidelberg, New York: Springer 1978

14. Taubes, C. H. : The existence of multi-monopole solutions to the static, SU (2) Yang-Mills-Higgs equations in the Prasad-Sommerfield limit. (to appear)

Communicated by J. Glimm

Received October 17, 1980 
